\title{
Performance Analysis of Low Loss Gas Filled Hollow Core Photonic Crystal Fiber (HC-PCF) for Transmission of THz Waves
}

\author{
Mohammad Tawfiq Hossain ${ }^{1}$, Md. RifatRayhan ${ }^{2}$ \\ ${ }^{1,2}$ Department of Electrical and Electronic Engineering, American International University-Bangladesh (AIUB), \\ Dhaka, Bangladesh
}

\begin{abstract}
A Kagome lattice hollow core photonic crystal fiber was filled by pumping pressurized inert gases (Argon, Krypton, Xenon) through the hollow core and wave guidance properties were observed for terahertz (THz) frequency. By using finite element method (FEM), effective material loss and confinement loss have been observed for different strut width, core diameter and different inert gases. Confinement of light has achieved through the hollow core for $\mathrm{THz}$ frequency. Lowest EML of 7.90x10-4 cm-1 is found for $5 \mu \mathrm{m}$ strut width and $800 \mu \mathrm{m}$ core diameter at $1 \mathrm{THz}$ frequency for Xenon gas pumped at 1000 bar pressure. Observation and findings of this paper will contribute in the ongoing research trends on $\mathrm{THz}$ waveguide.
\end{abstract}

Keywords: Confinement loss, effective material loss (EML), hollow core kagome lattice PCF, inert gas.

\section{Introduction}

Terahertz (THz) radiation is an electromagnetic radiation in a frequency interval from 0.1 to $10 \mathrm{THz}$. It is placed in between the infrared and microwave band [1]. Terahertz radiation can be found in any object with temperature more than $10 \mathrm{~K}$ [2]. THz radiation brought immense attention towards itself due to applications such as pharmaceutical drug testing [3], sensing, imaging, security, communications [4], medical diagnostics [5] etc. $\mathrm{THz}$ frequency range can transfer huge data files and contribute to the increment of communication data rate over the existing systems as well. As a result, THz generation [6] and detection [7] have been developed in the last decades. Existing THz systems largely depend on free space propagation since materials available for waveguides are very absorbent in this frequency band. But free space propagation of $\mathrm{THz}$ waves is not much efficient because due to loss during coupling, beam management difficulties and various other reasons. As a result, efficient waveguides for terahertz propagation had become necessary. Although, Circular metallic waveguides were introduced initially, it became obsolete for demonstrating too much loss [8]. Afterwards, hollow dielectric tubes with metal layer coating were reported [9], but they appeared to be bulky. Chen et al. used polyethylene as a guidance material [10], which has lower absorption loss. Recently, Topas [11], Polymethyl Methacrylate (PMMA) [12], Teflon [13] etc. are being used as primary choices for $\mathrm{THz}$ wave guiding. To reduce absorption loss further, research trends shifted to designing of guiding structure. Very recently photonic crystal fibers became popular among the researcher for their modification enabled property which helps controlling their behavior. A photonic crystal fiber is an optical fiber which obtains its waveguide properties from an arrangement of very tiny and closely spaced air holes which go through the whole length of fiber [14]. PCF can be of solid core, but it demonstrates huge material loss due to the absorption by the material that is present in the core [15]. To solve this problem, porous core PCF was introduced, which reduced the amount of material present in the core, thus reduced the material loss. The hollow core [16] PCF can improve the efficiency over the porous core PCFs as no material is present in the core. Although dry air has no material absorption in $\mathrm{THz}$ frequency range, non-linearity and dispersion in hollow core can be pacified by pumping pressurized gas through the hollow core of HC-PCF [17] [18].

In this paper, the hollow core of the kagome HC-PCF was filled with inert gases (argon, krypton and xenon) the properties such as effective material loss, confinement loss and other optical properties were observed.

\section{Design Procedure Of The Kagome Thz Hc-Pcf}

Fig. 1 shows the diagram of the Kagome lattice hollow core PCF. The cladding region of the PCF is formed with kagome lattice of micro structured air holes and the core is hollow, which would be filled with pressurized inert gas. TOPAS is used as background material to surround the kagome lattice of air holes and the hollow core. 


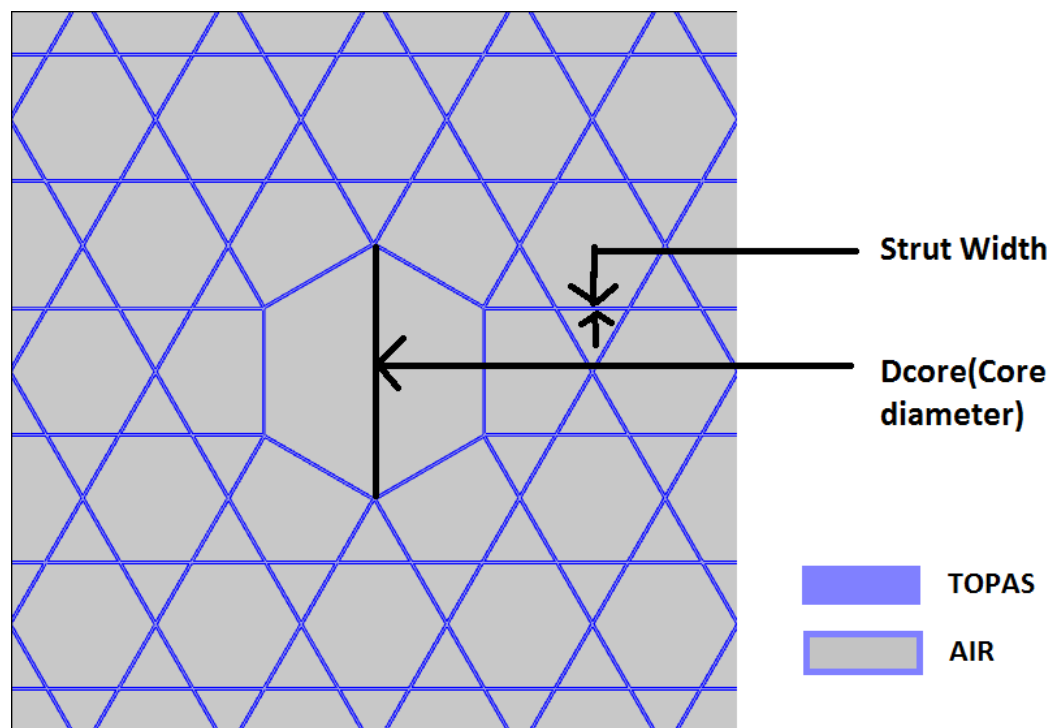

Figure 1: cross section of the hollow-core kagome lattice photonic crystal fiber

Fig. 1 itself describes the geometry of the kagome lattice hollow core PCF. And in the hollow core different inert gases could be pumped. The hollow core is of hexagonal shape and the core diameter (Dcore) is indicated in the figure as well.

\section{Results And Discussion}

Simulation is conducted using FEM based COMSOL Multiphysics to simulate the properties of the gas filled hollow core Kagome lattice HC PCF. To determine more accurate effective index, Sellmeier equation is used to calculate different gas' effective mode index as a function of wavelength, pressure and temperature [19].

$n 2(\lambda, p, T)=1+\frac{p}{P 0} \frac{T 0}{T}\left[\frac{B_{1} \lambda^{2}}{\lambda^{2}-C_{1}}+\frac{B_{2} \lambda^{2}}{\lambda^{2}-C_{2}}\right]$

In (1), numerical values of coefficients of inert gases, B1, B2, C1 and C2 at p0 =1 bar and T0 = 273k are found. Here, $\lambda$ is the operating wavelength, $\mathrm{p}$ is the gas pressure and $\mathrm{T}$ is the temperature.

The following expression is used for the estimation of the effective material loss (EML),

$\alpha_{e f f}=\frac{\alpha_{\text {mode }}}{\alpha_{\text {mat }}}=\frac{\left(\frac{\varepsilon 0}{\mu 0}\right)^{\frac{1}{2}} \int_{m a t} \eta \alpha_{m a t}|E|^{2} d A}{2 \int_{A l l} S z d A}$

The integration part of numerator in (2) is for the material section consisting of TOPAS and the denominator in (2) is for over all regions consists of whole material and air-hole region. $\varepsilon 0$ is the permittivity of the vacuum and $\mu 0$ is the permeability of the vacuum. Equation (2) also indicates the absorption loss ratio. Power flow in the direction of propagation has been calculated from pointing vector $\mathrm{Sz}$ which is consisted of the electric field component, $\mathrm{E}$ and the magnetic field component, $\mathrm{H}$.

$$
\mathrm{Sz}=0.5 \operatorname{Re}\left(\mathrm{ExH}^{*}\right)
$$

Confinement loss is actually the light restricting ability of the fiber in the core. It is calculated by the following equation,

$\alpha_{c}=\left(\frac{4 f}{c}\right) \operatorname{Im}\left(\eta_{e f f}\right)$

where, $\mathrm{f}$ is the frequency of light, and $\mathrm{c}$ is the velocity of light $(3 \times 108 \mathrm{~ms}-1)$. The imaginary part of the effective

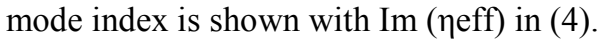

Fig. 2,3 and 4 demonstrate the effective material loss for different core width (Dcore) and $5 \mu \mathrm{m}, 6 \mu \mathrm{m}$, $7 \mu \mathrm{m}$ strut width at operating frequency of $1 \mathrm{THz}$. It is observed that the EML tends to reduce with the increase of core width (Dcore). 


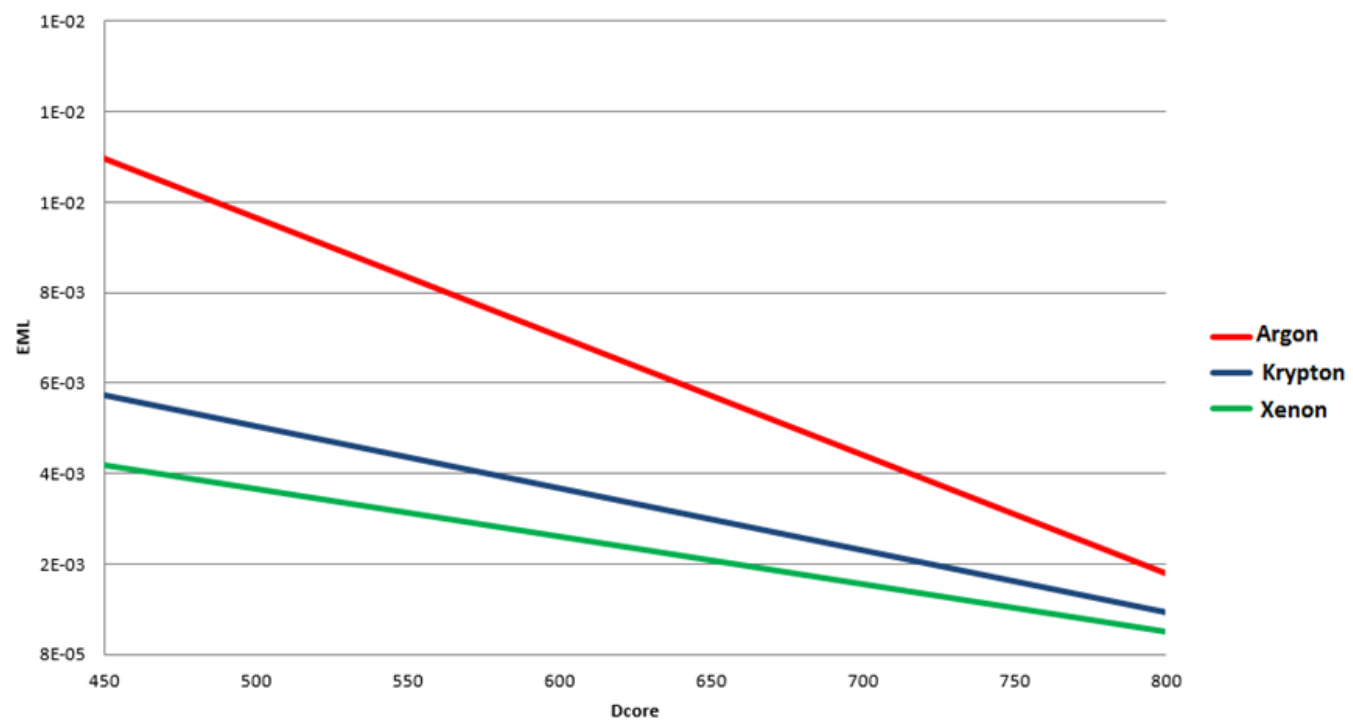

Figure 2: EML of argon, krypton and xenon for different Dcore at $1 \mathrm{THz}$ for $5 \mu \mathrm{m}$ strut width.

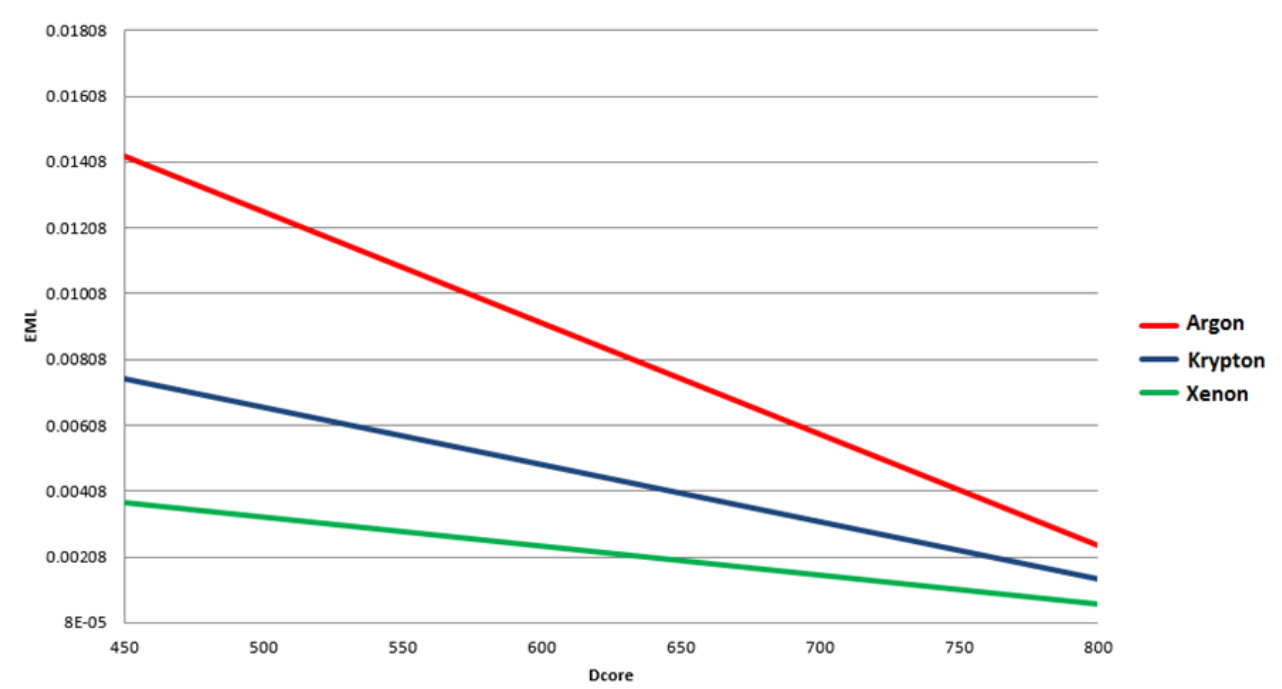

Figure 3: EML of argon, krypton and xenon for different Dcore at $1 \mathrm{THz}$ for $6 \mu \mathrm{m}$ strut width.

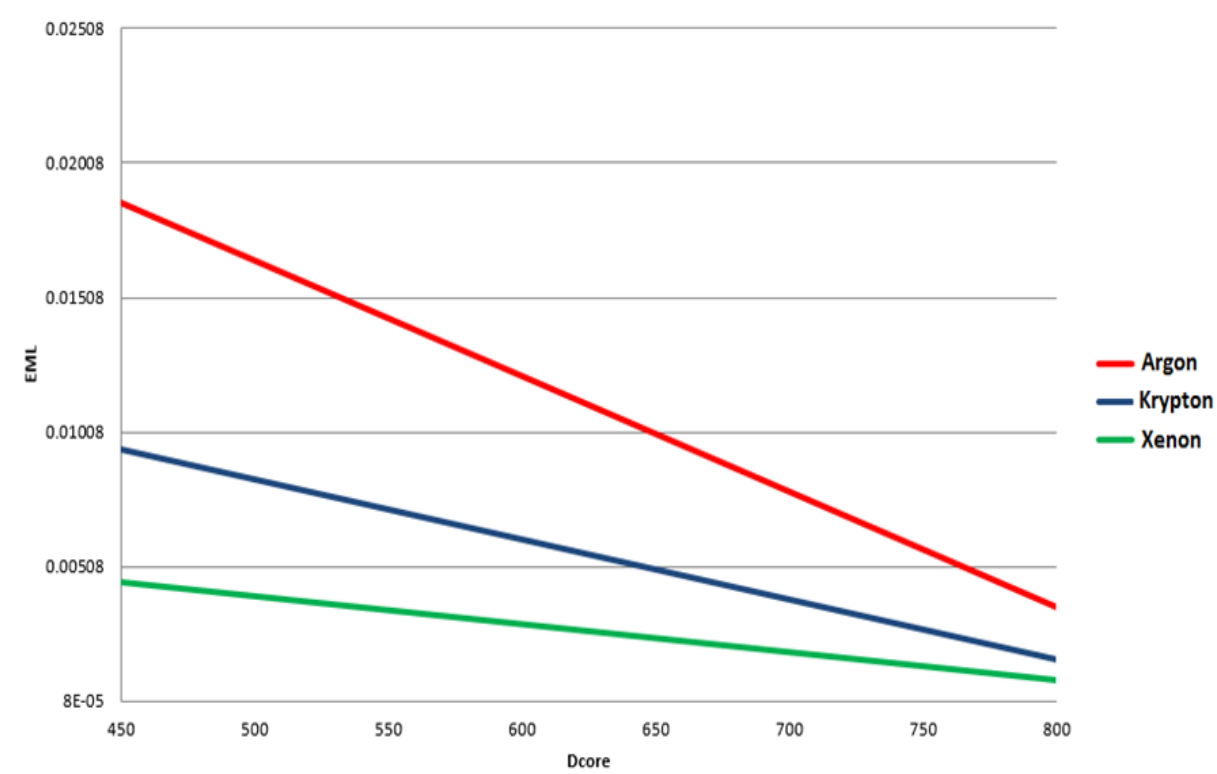

Figure 3: EML of argon, krypton and xenon for different Dcore at $1 \mathrm{THz}$ for $7 \mu \mathrm{m}$ strut width. 
From these graphs (Fig 2-4) it is also observed that Xenon filled hollow core demonstrates the lowest effective material loss.In the following table the confinement losses of the proposed HC-PCF are presented.

Table 1: Confinement Loss at $1 \mathrm{THz}$ for Different Strut Width,Dcore $(450,600,800 \mu \mathrm{m})$ and Inert Gases

\begin{tabular}{|c|c|c|c|c|c|c|c|c|c|}
\hline & \multicolumn{3}{|c|}{$5 \mu \mathrm{m}$ strut width } & \multicolumn{3}{|c|}{$6 \mu \mathrm{m}$ strut width } & \multicolumn{3}{|c|}{$7 \mu \mathrm{m}$ strut width } \\
\hline Gas & 450 & 600 & 800 & 450 & 600 & 800 & 450 & 600 & 800 \\
\hline $\mathrm{Ar}$ & $4.57 \times 10^{-5}$ & $3.76 \times 10^{-9}$ & $1.94 \times 10^{-13}$ & $5.40 \times 10^{-5}$ & $3.47 \times 10^{-10}$ & $1.04 \times 10^{-13}$ & $6.63 \times 10^{-5}$ & $8.21 \times 10^{-10}$ & $4.07 \times 10^{-13}$ \\
\hline $\mathrm{Kr}$ & $2.98 \times 10^{-11}$ & $1.44 \times 10^{-13}$ & $9.62 \times 10^{-14}$ & $4.55 \times 10^{-11}$ & $1.03 \times 10^{-13}$ & $2.12 \times 10^{-14}$ & $8.60 \times 10^{-11}$ & $3.01 \times 10^{-13}$ & $1.76 \times 10^{-13}$ \\
\hline $\mathrm{Xe}$ & $2.82 \times 10^{-12}$ & $4.12 \times 10^{-11}$ & $1.61 \times 10^{-14}$ & $6.25 \times 10^{-13}$ & $2.35 \times 10^{-13}$ & $4.24 \times 10^{-14}$ & $3.81 \times 10^{-12}$ & $3.09 \times 10^{-13}$ & $1.88 \times 10^{-13}$ \\
\hline
\end{tabular}

\section{Conclusion}

The hollow core of the kagome HC-PCF was filled by argon, krypton and xenon inert gases and optical properties were observed and optimized. It is found that the hollow core kagome PCF shows very low effective material loss of $7.90 \times 10^{-4} \mathrm{~cm}^{-1}$ when the hollow core is filled with xenon gas at 1000 bar pressure for $800 \mu \mathrm{m}$ core width and $5 \mu \mathrm{m}$ strut width.

\section{References}

[1] X.C.Zhang, Terahertz wave imaging: horizons and hurdles, phys. Med. Biol., vol. 47, no. 21, pp. 3667-3677, Oct., 2002.

[2] https://en.wikipedia.org/wiki/Terahertz_radiation, as of 15th march 2017.

[3] C.J. Strachan, P.F.Taday, D.A. Newnham, K.C. Gordon, J.A.Zeitler, M. Pepper, T.Rades, Using terahertz pulsed spectroscopy to quantify pharmaceutical polymorphism and crystallinity, J. Pharm. Sci. 94 (4) (2005) 837-846.

[4] M.M. Awad, R.A.Cheville, Transmission terahertz waveguide based imaging below the diffraction limit, Appl. Phys. Lett. 86 (22) (2005) 221107-1-221107-3.

[5] R.M. Woodward, V.P. Wallace, D.D. Arnone, E.H. Linfield, Pepper M, Terahertz pulsed imaging of skin cancer in the time and frequency domain, J. Biol. Phys. 29 (2/3) (2003) 257-261.

[6] R. Kohler, A.Tredicucci, F.Beltram, H.E. Beere, E.H. Linfield, A.G. Davies, D.A. Richie, R.C. Iotti, F. Rossi, Terahertz semiconductor-heterostructure laser, Nature 417 (6885) (2002) 156-159.

[7] N.E. Karpowicz, J. Chen, T. Tongue, X.C. Zhang, Coherent millimetre wave to mid-infrared measurements with continuous bandwidth reaching $40 \mathrm{THz}$, Electron. Lett. 44 (8) (2008) 544-545.

[8] K. Wang, D.M. Mittleman, Metal wires for terahertz wave guiding, Nature 432 (7015) (2004) 376-379.

[9] B. Bowden, J.A. Harrington, O. Mitrofanov,Silver/polystyrene-coated hollow glass waveguides for the transmission of terahertz radiation, Opt. Lett. 32 (20) (2007) 2945-2947.

[10] L.J. Chen, H.W. Chen, T.F. Kao, J.Y. Lu, C.K. Sun, Low-loss subwavelength plastic fiber for terahertz waveguiding, Opt. Lett. 31 (3) (2006) 308-310.

[11] K. Nielsen, H.K. Rasmussen, A.J. Adam, P.C. Planken, O. Bang, P.U. Jepsen, Bendable, low-loss Topas fibers for the terahertz frequency range, Opt. Exp. 17 (10) (2009) 8592-8601.

[12] C.S. Ponseca Jr., R. Pobre, E. Estacio, N. Sarukura, A. Argyros, M.C. Large, M.A. van Eijkelenborg, Transmission of terahertz radiation using a microstructured polymer optical fiber, Opt. Lett. 33 (9) (2008) 902-904.

[13] M. Goto, A. Quema, H. Takahashi, S. Ono, N. Sarukura, Teflon photonic crystal fiber as terahertz waveguide, Jpn. J. Appl. Phys. 43 (2B) (2004) L317-L319. pt. 2.

[14] P.St.J. Russell,Photonic-Crystal Fibers, J. Lightwave Technol. 24, 4729-4749 (2006).

[15] M. Goto, A. Quema, H. Takahashi, S. Ono, N. Sarukura, Teflon photonic crystal fiber as terahertz waveguide, Jpn. J. Appl. Phys. 43 (2B) (2004) L317-L319. pt. 2.

[16] G. Humbert, J.C. Knight, G. Bouwmans,P.St.J. Russell, D. Williams, P. Roberts, and B. Mangan, Hollow core photonic crystal fibers for beam delivery, Opt. Express, vol. 12, no. 8, pp. 1477-1484, Apr. 2004.

[17] M. Azhar, G.K.L. Wong, W. Chang, N.Y. Joly, and P.St.J. Russell, High pressure gases in hollow core photonic crystal fiber: A new nonlinear medium, October 2012.

[18] J.C. Travers, W. Chang, J. Nold, N.Y. Joly, P.St.J. Russell, Ultrafast nonlinear optics in gas-filled hollow-core photonic crystal fibers [Invited], J. Opt. Soc. Am. B 28, A11-A26 (2011).

[19] A. Börzsönyi, Z. Heiner, M.P. Kalashnikov, A.P. Kovács, K. Osvay, Dispersion measurement of inert gases and gas mixtures at 800 nm, Applied optics vol. 47, no. 27 pp: 4856-4863, September 2008. 